\title{
Locating Delamination in Composite Laminated Beams Using the Zero-Order Mode of Lamb Waves
}

\author{
Yaolu Liu, Alamusi, Jinhua Li, Huiming Ning, \\ Liangke Wu, Weifeng Yuan, Bin Gu and Ning Hu
}

Additional information is available at the end of the chapter

http://dx.doi.org/10.5772/49991

\section{Introduction}

To improve the safety and reliability of various engineering structure, it is essential to develop efficient techniques for non-destructive damage detection or structural health monitoring. Lamb wave can travel a long distance in plate-like and shell-like structures made of materials even with high attenuation ratio (e.g. Carbon Fibre/Epoxy Polymer composites). To take this advantage, many researchers have recently explored the possibility of using Lame waves for damage identification [1]. To date, many developed Lamb wavebased techniques are generally based on so called two-stage prediction models by which the difference in the signals between a defective structure and a benchmark (intact structure) can be evaluated. Then, the residual error is easy to be defined no matter what information extracted from the signals is used, such as the information in time domain [2, 3] or frequency domain [4,5]. Therefore, a benchmark or baseline signal is essential for the detection, which is very reliable and suitable for monitoring the propagation of damage. Also, tremendous efforts have been put to the delamination identification, which could be treated as a problem of inverse pattern recognition using calibrated numerical methods such as artificial neural network [6]. The interaction between Lamb waves and delamination has also been investigated numerically and theoretically [7-9]. However, the complex wave scattering phenomenon in a delamination area has not been clearly understood in these studies.

In this chapter, a technique for delamination identification in laminated composites using the zero-order mode of Lamb waves, i.e., S0 mode and A0 mode, without referring to the baseline data, is described. Through measuring the propagation speed of a wave and the traveling time of a reflected wave from the delamination, the delamination position can be 
accurately identified. Moreover, to understand the complex interaction of Lamb waves with a long delamination damage, the numerical simulations have been carried out.

\section{Detective technique without the baseline data}

\subsection{Experiments and numerical analyses}

In general, compared with $\mathrm{S} 0$ mode, the attenuation of A0 mode in structures is more severe. Here, S0 mode corresponds to an axial deformation mode while A0 mode corresponds to a flexural deformation mode. Therefore, it should be paid more attention to the detective capability of both modes for a delamination damage in the different interfaces along the thickness direction. Moreover, the experimental setup of S0 mode is slightly different with that of A0 mode.

\subsubsection{Materials and experimental procedure}

For S0 mode, as shown in Fig. 1, a CFRP laminated composite beam of stack sequence of $\left[0_{10} / 90_{12} / 0_{10}\right]$ was used. An artificial delamination damage with different lengths for several cases, i.e., $30 \mathrm{~mm}, 20 \mathrm{~mm}$ and $10 \mathrm{~mm}$, respectively, was intentionally created at the interface between the 10th and 11th plies by inserting a Teflon film with a thickness of $25 \mu \mathrm{m}$. A PZT actuator was attached on the top surface of the left end of the beam. The PZT actuator had a diameter of $10 \mathrm{~mm}$ and a thickness of $0.5 \mathrm{~mm}$. In this case, the generated waves from the actuator merged into the reflected waves from the left end of beam, which leads to a simpler signal. And the same PZT unit was used as a sensor to pick up the reflected wave from the delamination damage. Because the propagation speed of S0 mode is much higher than that of A0 mode, confirmed as 4 times higher in the used composites from our testing, it is expected that in a specified time domain, no A0 mode will be collected due to its slow speed, making the analysis of S0 modes much easier.

For the A0 mode, a similar composite beam was used, as shown in Fig. 2. Two kinds of stack sequence of the laminated beam were used, i.e., [010/9012/010] and $\left[0_{12} / 0_{4} / 0_{4} / 0_{12}\right]$. Note that $\left[0_{12} / 0_{4} / 0_{4} / 0_{12}\right]$ is a unidirectional laminate, and it can be simply expressed as $\left[0_{32}\right]$. To simplify the description of delamination later, this expression is used. Like the case of S0 mode, a delamination damage with different lengths, i.e., $30 \mathrm{~mm}, 20 \mathrm{~mm}$ and $10 \mathrm{~mm}$, was intentionally created at the interface between two plies by inserting a Teflon film with a thickness of $25 \mu \mathrm{m}$. The distance between the center of the delamination and the left end of beam is $790 \mathrm{~mm}$. To generate the A0 mode, two actuators were attached on the top and bottom surfaces of the beam with applied out-of-phase voltages because the difference of signals in the two actuators can produce the pure $A_{0}$ mode, as shown in Fig. 2. Although this arrangement can generate a relatively pure A0 mode, there are still reflections in S0 mode due to the mode change caused by the scattering between the Lamb waves and the delamination. To pick up the pure $A_{0}$ mode, two PZT sensors were attached. We know that two components of A0 mode in the two sensor signals are of the electrical charges of opposite signs, and two components of S0 mode in the two sensor signals are of the electrical charges of same signs. Naturally, the difference of 
two signals can yield the pure A0 mode. In experiments, an excitation signal in the following Eq. (1), was adopted to generate S0 or A0 mode.

$$
P(t)=\left\{\begin{array}{c}
0.5[1-\cos (2 \pi f t / N) \sin (2 \pi f t)], \quad t \leq N / f \\
0, \quad t>N / f
\end{array}\right.
$$

where $f$ is the central frequency in $\mathrm{Hz}$ and $N$ is the number of sinusoidal cycles within a pulse. In this experiment, the signal with $f=100 \mathrm{kHz}$ (Fig. 1 for S0 mode) or $f=50 \mathrm{kHz}$ (Fig. 2 for A0 mode) and $N=5$ was used and the electrical voltage on the actuator was $10 \mathrm{~V}$. The material properties of PZT are listed in Table 1.

Without the baseline data, it is still easy to estimate the arrival of the reflections from the beam boundaries, provided that the wave propagation speed and the dimensions of the beam are known. Then, if the reflected signal from the delamination is not overlapped with the reflections from the boundaries, it can be simply collected by the sensor. Therefore, it is still a technical challenge when the delamination is located close to the beam boundaries and the reflected signal of the delamination is completely overlapped with the reflections from the boundaries.

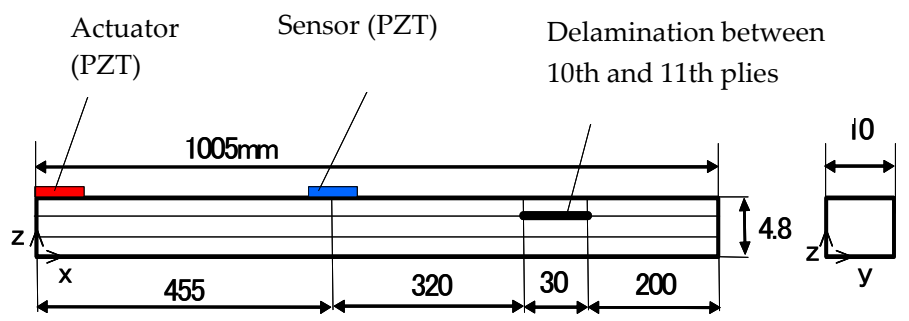

Figure 1. Schematic view of models for experiments using $\mathrm{S} 0$ mode (unit: $\mathrm{mm}$ )

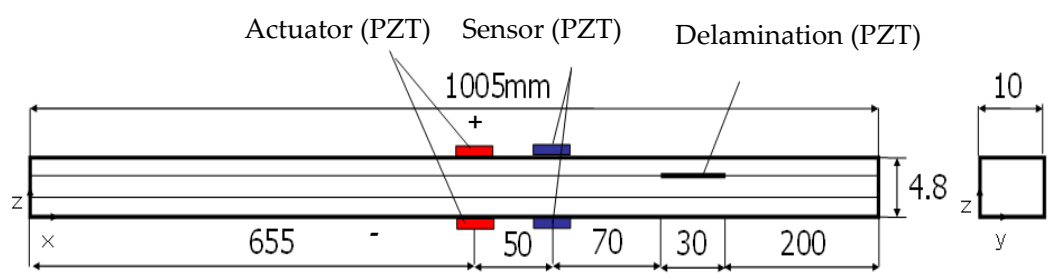

Figure 2. Schematic view of models for experiments using A0 mode (unit: $\mathrm{mm}$ )

\section{Material properties}

PZT

$$
\begin{gathered}
\mathrm{E}_{11}=62 \mathrm{GPa}, \mathrm{E}_{33}=49 \mathrm{GPa}, \mathrm{d}_{33}=472 \mathrm{pC} / \mathrm{N}, \mathrm{d}_{31}=-210 \mathrm{pC} / \mathrm{N}, \rho=7500 \mathrm{~kg} / \mathrm{m}^{3} \\
\mathrm{E}_{11}=115 \mathrm{GPa}, \mathrm{E}_{22}=\mathrm{E}_{33}=9 \mathrm{GPa}, \mathrm{G}_{12}=\mathrm{G}_{23}=5.5 \mathrm{GPa}, \mathrm{G} 13=3 \mathrm{GPa}, \\
v_{12}=v_{13}=0.3, v_{23}=0.45, \rho=1600 \mathrm{~kg} / \mathrm{m}^{3}
\end{gathered}
$$

Table 1. Material properties of PZT and CFRP lamina 


\subsubsection{Finite element analysis procedure}

To explore the wave propagation in laminates with a long delamination case, a threedimensional 8-noded brick hybrid element proposed by the authors [10] and the explicit time integration algorithm were used in the finite element simulations without considering the dynamic contact effects like those in some previous studies [11-13]. For the delamination area, one node used on the intact interface along the through-thickness direction, and double nodes were used on the delamination interface, i.e., one belongs to the elements of upper delaminated portion, and the other belongs to the elements of lower delaminated portion. The contact effects in the delamination area were neglected. The same signal stated in Eq. (1) was also used here. Furthermore, the actuator and the sensor were discretized using 3D brick elements. If an electrical field is applied on the actuator, the tensional or compressive strains will be generated in it from a proper relation, which connects the applied voltage and the generated internal strains. The induced stresses from the generated strains can be used to calculate the elemental nodal forces, which form axial force and bending moment in the beam simultaneously. Then, both $\mathrm{S} 0$ and $\mathrm{A} 0$ modes can be automatically generated. The material properties of CFRP are shown in Table 1.

\subsection{Results of $S 0$ mode}

\subsubsection{Experimental analysis}

Firstly, to evaluate the wave propagation speed, an intact beam with two sensors attached, was employed. The distance between the two sensors was $420 \mathrm{~mm}$. By using the wavelet transformation technique [14], the arrival times of the incident waves to the two sensors were determined and the wave propagation speed of $S 0$ mode at $f=100 \mathrm{kHz}$ was estimated as $6210 \mathrm{~m} / \mathrm{s}$. To verify the experimental results, the theoretical wave speed of the S0 mode was estimated based on the transfer matrix method [15]. The calculated speed was $6467 \mathrm{~m} / \mathrm{s}$ if the material properties of CFRP were taken as the values of Table 1 . On the other hand, the corresponding propagation speed of A0 mode was estimated as $1506 \mathrm{~m} / \mathrm{s}$, much lower that the S0 mode. The higher theoretical wave speed of S0 mode may be due to the transfer matrix method used here, which is actually for an infinite plate. For a beam with a finite width, the influences of Poisson's ratio and boundary conditions are expected.

A comparison of the signals from an intact beam and a delaminated one (a $30 \mathrm{~mm}$ delamination damage) for the case in Fig. 1 is shown in Fig. 3. It is clear that there is a reflected wave in the signals from the delaminated beam, which is located between the incident and the reflected waves. However, no clear reflected wave could be detected when the delamination length was reduced to $10 \mathrm{~mm}$. No A0 mode can be observed in Fig. 3, since its incident wave is not fast enough to reach the sensor within $300 \mu \mathrm{s}$. For a signal with $f=100$ $\mathrm{kHz}$ and $N=5$, the minimum detectable length of the delamination was $20 \mathrm{~mm}$, which is about $1 / 3$ of the wavelength of the S0 mode at $100 \mathrm{kHz}$. It is reasonable to assume that a higher excitation frequency may have the benefit of detecting smaller delamination cases. Once the arrival time of a reflected wave from the delamination is determined, the difference between the arrival times of the incident and reflected waves can be used to 
detect the delamination position, as shown in Fig. 4. For the $20 \mathrm{~mm}$ long delamination, a slightly higher error in the delamination position is observed. The reason is the overlapping between the reflected signals from the delamination and those from the right end of the beam, causing an increased error in determination of the arrival time of a reflected wave from the delamination.

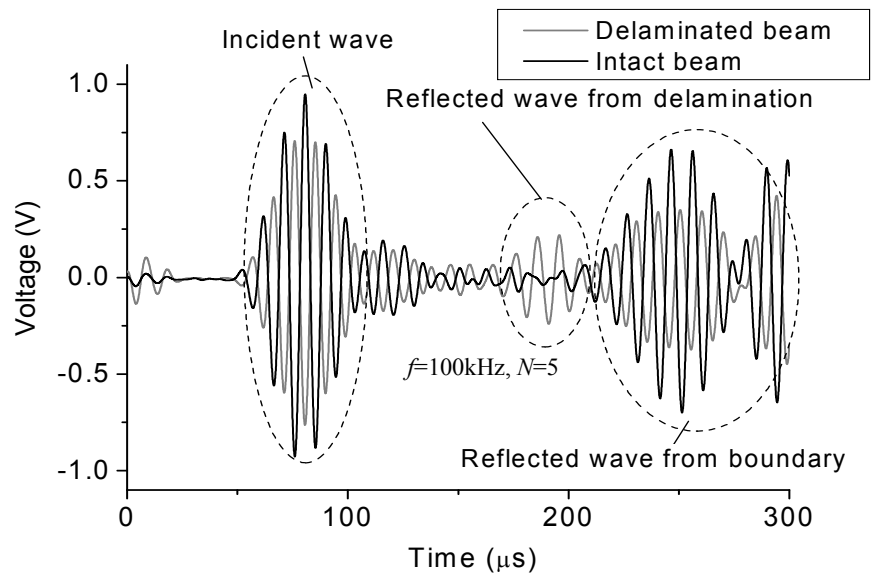

Figure 3. Comparison between signals of delaminated and intact beams (a $30 \mathrm{~mm}$ delamination damage between the 10th and the 11th plies)

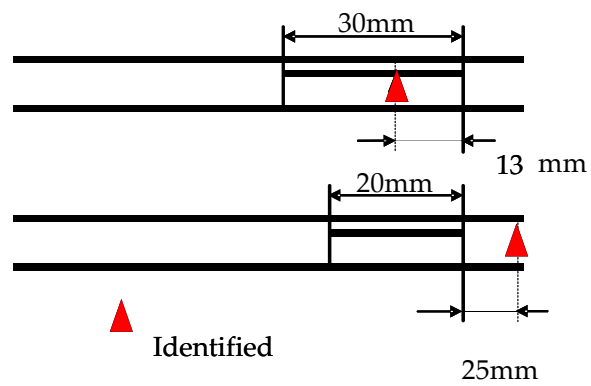

Figure 4. Delamination positions identified experimentally 


\subsubsection{Numerical simulation}

A comparison between the experimental result and numerical simulation for the case of Fig. 1 is shown in Fig.5. A good agreement between two results is observed. For convenience, the amplitude of the first arrival S0 mode in the simulated waves was calibrated using the experimental data. The attenuation coefficients of the CFRP in the numerical model were determined by matching the amplitudes of the reflected waves from the right end of the beam to the experimental data. When the actual sensor thickness, i.e., $0.5 \mathrm{~mm}$, was used in numerical simulations, a small reflected wave from the sensor could be observed immediately next to the incident wave. This was also confirmed by the experimental result. Corresponding to a $0.05 \mathrm{~mm}$ sensor thickness, however, no obvious reflection from the sensor could be observed in the numerical simulation. Therefore, the sensor thickness of 0.05 $\mathrm{mm}$ was eventually chosen in the simulations. Note that the selection of sensor thickness does not affect the simulation results as the calculated amplitudes will be calibrated using the experimental data. Similar to the experimental results, the numerical simulation showed that the reflected wave from the $10 \mathrm{~mm}$ delamination was very weak.

To understand the effect of delamination position along the through-thickness direction on the propagation of a Lamb wave, a $30 \mathrm{~mm}$ delamination damage was created in the midplane of the laminated beam, i.e., between the 16th and the 17th plies. With the signal of $f=80$ $\mathrm{kHz}$ and $N=5$, the comparison between the numerical and experimental results is shown in Fig.6. In Fig.6, no obvious reflected waves from the delamination can be observed. This finding is consistent with the work of Guo et al. [7]. They showed that the delamination at the positions of zero shear stress along the through-thickness direction, such as the midplane of a beam, had no effect on Lamb wave propagation in a S0 mode.

To examine the capability of the numerical simulation to identify the location of delamination, the length of delamination L was increased from 30 to $90 \mathrm{~mm}$. The length of the beam was also increased up to $1500 \mathrm{~mm}$. Under a condition of $f=100 \mathrm{kHz}$ and $N=5$, the propagation speed of S0 mode was firstly calculated in an intact beam with two sensors. The estimated propagation speed was $6260 \mathrm{~m} / \mathrm{s}$, which is very close to the experimental result. Based on this speed, the numerically identified positions for various delamination lengths are shown in Fig. 7(a). It is surprising to note that all predicted positions are beyond the right end of the delamination. It has been demonstrated that a higher propagation speed of S0 mode in the delaminated $0^{\circ}$ layer can make the predicted delamination positions behind the actual delamination [16, 17]. From the FEM simulations, the estimated speed of S0 mode in the $0^{\circ}$ delaminated layer was $8483 \mathrm{~m} / \mathrm{s}$. If we use this speed in the delaminated region only (in the intact region, we still use $6260 \mathrm{~m} / \mathrm{s}$ ), with the known left end of the delamination, the predicted delamination positions or the reflected positions of S0 mode are shown in Fig. 7(b). Compare it with Fig. 7(a), we can find that there is no obvious difference in the delamination positions for short delamination cases, e.g. 30 and $50 \mathrm{~mm}$. However, for a longer delamination case (e.g., 70 and $90 \mathrm{~mm}$ ), an increased deviation from the actual delamination is observed when $8483 \mathrm{~m} / \mathrm{s}$ was used in the prediction. It implies that the use of the actual wave speed of the delaminated layer cannot improve the prediction and a reflected wave with observable intensity is normally from the right end of the delamination. 


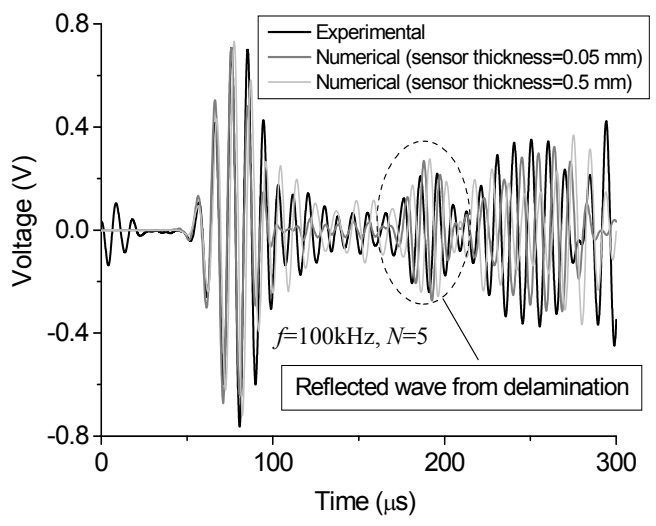

Figure 5. Comparison of experimental and numerical results for a $30 \mathrm{~mm}$ delamination damage between the $10^{\text {th }}$ and the 11 th plies

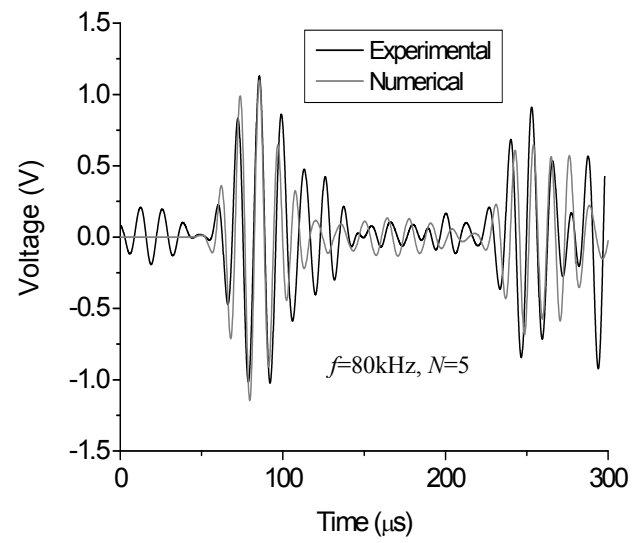

Figure 6. Comparison of experimental and numerical results for a $30 \mathrm{~mm}$ delamination damage at the mid-plane between the 16th and the 17th plies 


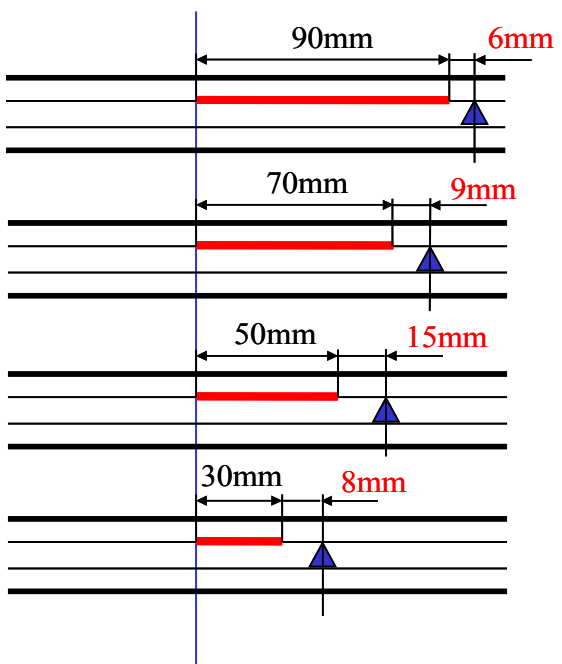

$\Delta:$ Identified

(a) Results using $6260 \mathrm{~m} / \mathrm{s}$

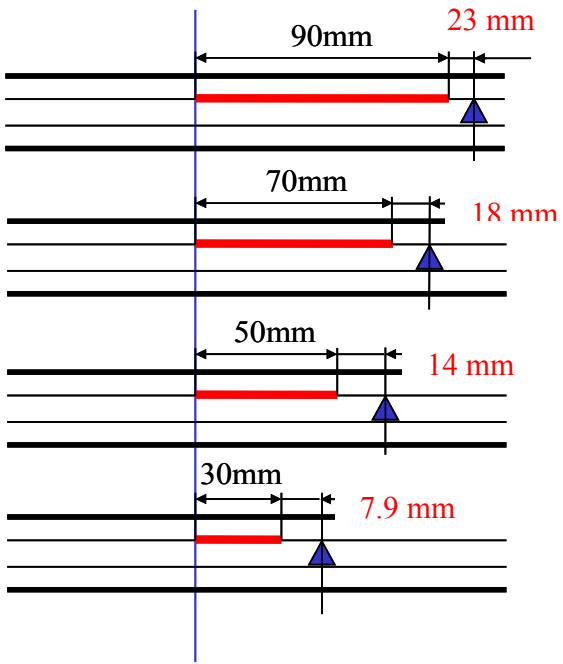

$\Delta:$ Identified

(b) Results using $8483 \mathrm{~m} / \mathrm{s}$ in $0^{\circ}$ delaminated layer

Figure 7. Numerical identification of positions of various delamination cases

\subsection{Results of A0 mode}

To confirm the effectiveness of present excitation and sensing techniques for A0 mode (Fig. 2 ), numerical simulation was firstly conducted for the laminate with $\left[0_{10} / / 90_{6} / 90_{6} / 0_{10}\right]$ and 30 mm delamination under a condition of $f=50 \mathrm{kHz}$ and $N=5$. Note that "//" denotes the position of the delamination. The signals from upper and lower sensors are plotted in Fig. 8(a). From the first wave packet, it can be observed that the pure A0 mode can be excited by two actuators with the out-of-phase applied voltages and two signals with a mutual phase difference of $180^{\circ}$ are generated. Also, after the incident wave, the direct reflected signal from the delamination can be clearly observed. As mentioned before, the reflected signals from the delamination may include two modes, i.e., S0 mode and A0 mode as marked in Fig. 8(a). It can be explained by the wave mode change when the incident A0 mode interacts with the delamination. In Fig. 8(a), the reflections of the transmitted waves from the boundary can be identified, which also contain the S0 mode and A0 mode. The difference of two signals between the two sensors in Fig. 8(a) is shown in Fig. 8(b). It is clear that the S0 mode has been completely removed, and only pure A0 mode exists.

The results of the laminate $\left[0_{10} / / 90_{6} / 90_{6} / 0_{10}\right]$ with $30 \mathrm{~mm}$ and $10 \mathrm{~mm}$ delamination damages are shown in Fig 9. Sampling time for both numerical and experimental results was $1.0 \times 10^{-4}$ ms. For the delamination of the lengths of $30 \mathrm{~mm}$ and $10 \mathrm{~mm}$, the reflections from the delamination can be clearly identified. In Figs. 9(a) and 9(b), it is interesting to note that the reflection signal from the $10 \mathrm{~mm}$ delamination is stronger than that from the $30 \mathrm{~mm}$ delamination. The reason may be that the reflections from the two ends of delamination are 
more easily overlapped in a shorter delamination case, e.g., the $10 \mathrm{~mm}$ one, resulting in a higher total reflection. Therefore, the intensity of the reflection from the delamination does not certainly depend on the length of the delamination. When the delamination is located on the mid-plane of the laminates, i.e., $\left[010 / 90_{6} / / 90_{6} / 0_{10}\right]$, the results are shown in Fig. 10. Similar to Fig. 9, the reflection can be identified clearly. Also, the reflection from $10 \mathrm{~mm}$ delamination is still stronger than that of $30 \mathrm{~mm}$. For the short delamination, e.g., smaller than $30 \mathrm{~mm}$, reflections from the two ends of the delamination should be overlapped if we consider the duration time of a 5 cycle signal $(N / f$ with the number of cycles $N$ and wave central frequency $f$ ), the length of delamination and the travelling speed of A0 mode. By comparing Figs. 10(a) and 10(b), it can found that the duration of the reflected signal from the $30 \mathrm{~mm}$ delamination is obviously longer than that of the $10 \mathrm{~mm}$ delamination although its amplitude is smaller. It implies that the overlapping degree of two reflections from the two ends of the $30 \mathrm{~mm}$ delamination is lower than that of the $10 \mathrm{~mm}$ delamination, which leads to the lower intensity reflection from the $30 \mathrm{~mm}$ one. For the laminates with $\left[0_{12} / 0_{4} / / 0_{4} / 0_{12}\right]$ and $\left[0_{12} / / 0_{4} / 0_{4} / 0_{12}\right]$, the anti-symmetric A0 mode still works very well and the reflection from the delamination can be clearly identified. Only the difference of two sensor signals for the case of $\left[0_{12} / 0_{4} / / 0_{4} / 0_{12}\right]$ with $10 \mathrm{~mm}$ delamination is illustrated in Fig. 11.

From Fig. 9 to Fig. 11, a good agreement between experimental and numerical results is observed. For convenience, the amplitude of the first arrival A0 mode in the simulated waves was calibrated using the experimental data. The attenuation coefficients of the CFRP in the numerical model were determined by matching the amplitude of the reflected waves from the right end of the beam to that of experimental data. Naturally, the material properties of CFRP in Table 1 were also adjusted slightly within a reasonable range to match the numerical results to the experimental ones.

To determine the wave propagation speed of A0 mode, two sets of distant sensor pairs were attached on an intact laminated beam. The wave speed was determined from the distance and the difference of arrival times between the two sets of sensor pairs. For the laminate with [010/906/906/010], the experimental and numerical wave speeds were $1555 \mathrm{~m} / \mathrm{s}$ and $1470 \mathrm{~m} / \mathrm{s}$, respectively. For the case of $\left[0_{12} / 0_{4} / 0_{4} / 0_{12}\right]$, the experimental and numerical wave speeds were $1723 \mathrm{~m} / \mathrm{s}$ and $1616 \mathrm{~m} / \mathrm{s}$, respectively. From these results, it can be found that the wave speed of $\left[0_{12} / 0_{4} / 0_{4} / 0_{12}\right]$ is only slightly higher than that of $\left[010 / 90_{6} / 90_{6} / 0_{10}\right]$. The reason is that the wave speed of A0 mode is determined by the bending stiffness of laminates, which is dominated by the fibre orientation of outer layers near top and bottom surfaces of laminates. In both cases, $0^{\circ}$ degree layers are located near the two surfaces of laminates, which results in the comparatively small difference of wave speeds in both cases. For the case of $\left[010 / 90_{6} / 90_{6} / 0_{10}\right]$, if $90^{\circ}$ degree layers were located near the two surfaces of laminates, it would be a completely different story.

With the knowledge of the wave speeds, the delamination positions for various situations were evaluated, as shown in Table 2. The distances between identified positions and the actual centers of delamination are listed. In this table, the negative values denote that the identified positions are located on the left side of the delamination centers, and positive values represent that the identified positions are on the right side of the delamination centers. In this table, it is clear that the delamination positions in almost all laminates have been 
successfully detected compared with the beam length. For the laminate with $\left[0_{10} / / 90_{6} / 90_{6} / 0_{10}\right]$ and $20 \mathrm{~mm}$ delamination, the reflection is not very clear due to strong noises in experiments. As a result, only numerical estimation is presented in this table. The similar case can be found in the laminate with $\left[0_{12} / 0_{4} / / 0_{4} / 0_{12}\right]$ and $30 \mathrm{~mm}$ delamination, as shown in this table.

In summary, unlike S0 mode, A0 mode can be used for different applications. However, the amplitude of reflection from delamination may not depend on the length of the delamination. The amplitude of reflection from short delamination is higher than that from long delamination due to overlapping of reflections from the ends of short delamination. The disadvantage of A0 mode is the short travelling distance due to its high attenuation in CFRP materials. Therefore, if possible, both the actuators and sensors should be placed close to the potential damage sites.

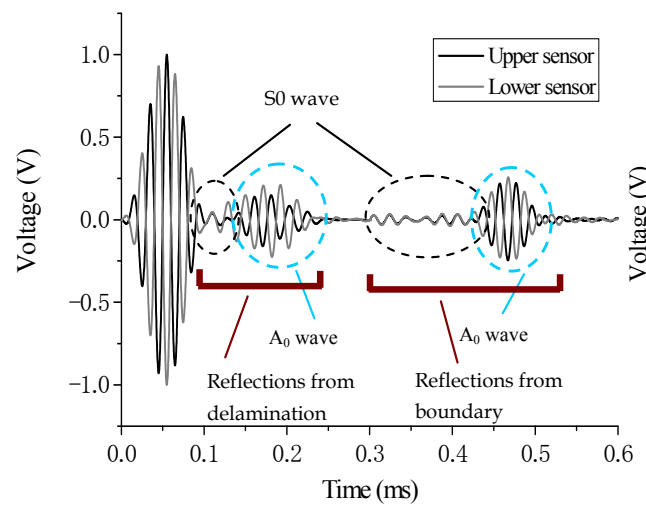

(a) signals of upper and lower sensors

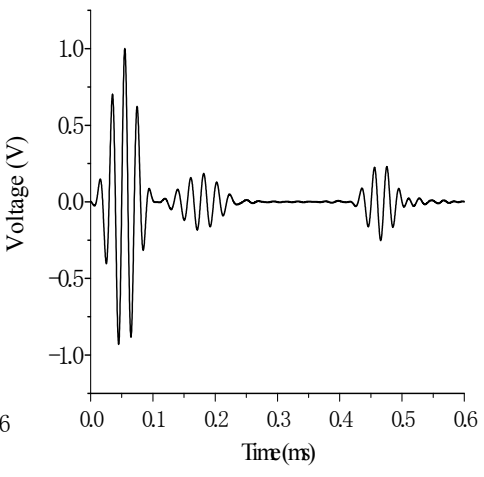

(b) difference of signals of upper and lower sensors

Figure 8. FEM numerical results for [010//906/906/010] (delamination length: $30 \mathrm{~mm}$ )

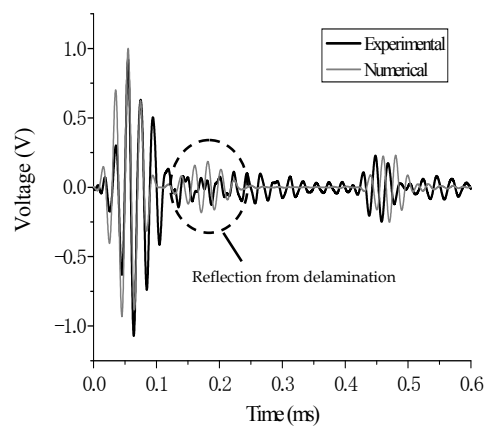

(a) delamination length: $30 \mathrm{~mm}$

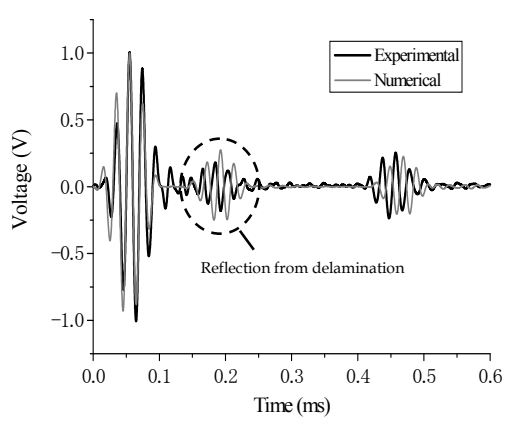

(b) delamination length: $10 \mathrm{~mm}$

Figure 9. Comparison of numerical and experimental results for $\left[010 / / 90_{6} / 90_{6} / 0_{10}\right]$ 

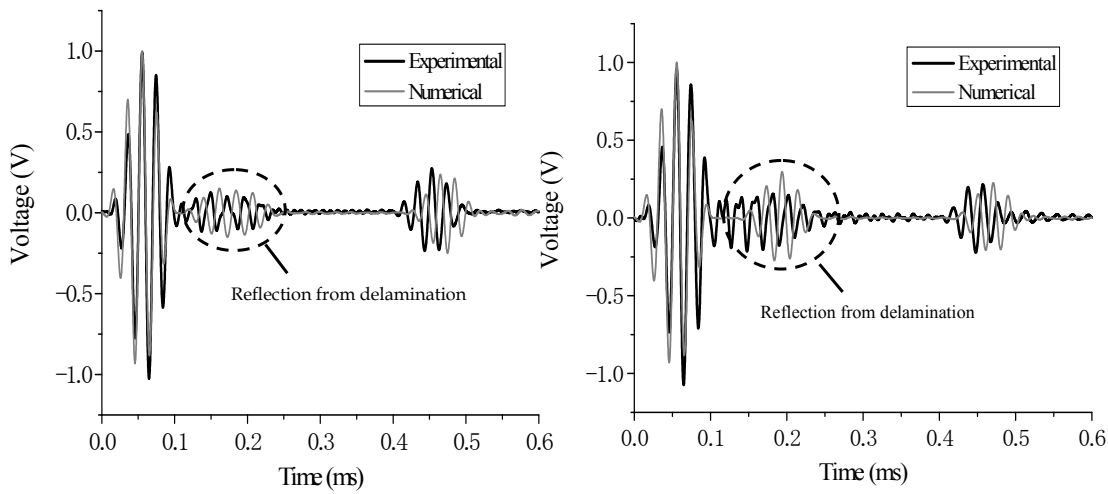

Figure 10. Comparison of numerical and experimental results for $\left[010 / 90_{6} / / 90_{6} / 0_{10}\right]$

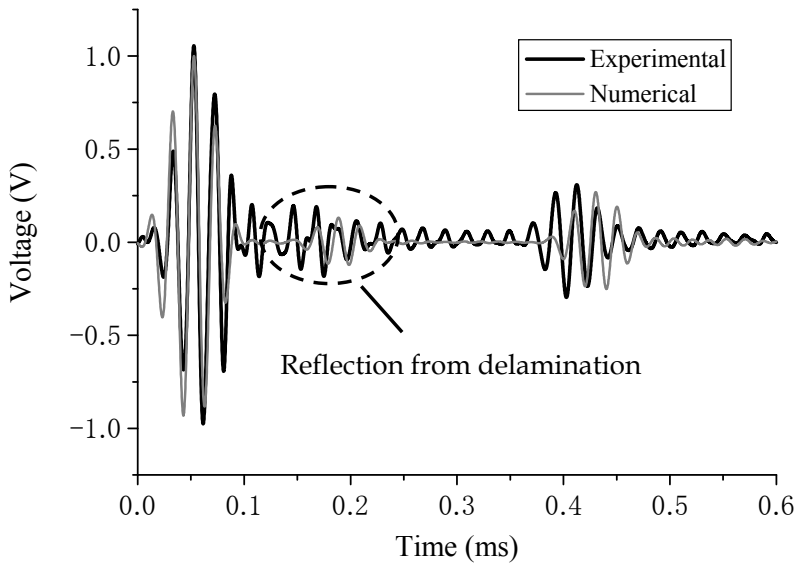

Figure 11. Comparison between experimental and numerical results for a $10 \mathrm{~mm}$ delamination $\left(\left[012 / 0_{4} / / 0_{4} / 0_{12}\right]\right)$

\begin{tabular}{||c|c||c|c|c|c|}
\hline \hline \multicolumn{2}{|c|}{ Unit $(\mathrm{mm})$} & {$[0 / 90 / / 90 / 0]$} & {$[0 / / 90 / 90 / 0]$} & {$[0 / 0 / / 0 / 0]$} & {$[0 / / 0 / 0 / 0]$} \\
\hline \multirow{2}{*}{$30 \mathrm{~mm}$} & Num. & -11.0 & 4.0 & 0.0 & 18.0 \\
\cline { 2 - 6 } & Exp. & -19.0 & 39.0 & & -11.0 \\
\hline \multirow{2}{*}{$20 \mathrm{~mm}$} & Num. & -12.0 & -5.0 & -14.0 & 18.0 \\
\cline { 2 - 6 } & Exp. & 9.0 & -11.0 & 4.0 \\
\hline \multirow{2}{*}{$10 \mathrm{~mm}$} & Num. & 2.0 & 2.0 & 1.0 & 1.0 \\
\cline { 2 - 6 } & Exp. & 2.0 & -2.0 & 10.0 & -17.0 \\
\hline
\end{tabular}

Table 2. Identified delamination positions for various cases 


\section{Interaction between Lamb waves and delamination}

\subsection{S0 mode}

To gain a better understanding of this complex interaction, a beam with a length of $1500 \mathrm{~mm}$ and a $200 \mathrm{~mm}$ long delamination damage was examined using the numerical simulation under a condition of $f=100 \mathrm{kHz}$ and $N=5$. The very long delamination was chosen for obtaining the separated reflected signals from the two ends of the delamination. The transverse deflections at various mesh points on the top surface of the beam were used and the subtraction between the wave signals from the intact and the delaminated beams was done to amplify the scattered wave signals from the delamination. Fig. 12 shows the detail information of the scattered wave signals from the delamination at different time domains. The typical sensor signal for accurately evaluating the arrival times of various waves is shown in Fig. 13.

At the time domain of $139 \mu \mathrm{s}$, the S0 mode arrives at the left end of the delamination and then reflects from or transmits from the left end, as shown in Fig. 12(a). At $182 \mu \mathrm{s}$, the S0 mode passes through the right end of the delamination. Fig. 12(b) shows two separate reflected modes, i.e., A0 and S0 from the left end of the delamination. In principle, it is easy to distinguish a $\mathrm{S} 0$ from an A0 mode by the propagation speed and wave length. However, the amplitude of the reflected S0 mode is smaller than that of the A0 mode. This can be explained by the fact that the bending deformation in a laminated beam is much higher as compared to the axial deformation. Also, when the S0 mode travels through the left end of delamination, a new transmitted A0 mode is generated as shown in Fig. 12(b). The reflected waves from the right end of the delamination, consisted both $\mathrm{A} 0$ and $\mathrm{S} 0$ modes, can be also observed in Fig. 12(b). Corresponding to $236 \mu \mathrm{s}$, the incident A0 mode from the actuator does not reach the sensor, and the reflected S0 mode from the left side of the delamination has already passed through the sensor. However, at the same time point in Fig. 13, this reflected S0 mode cannot be detected by the sensor due to its small amplitude. Also, the reflected $\mathrm{S} 0$ mode from the right end of the delamination, which is surpassing the reflected A0 mode from the left end of the delamination, can be observed, as shown in Fig. 12(c). A new transmitted A0 mode and the transmitted S0 mode from the right end of the delamination can be clearly observed, Fig. 12(c). At $303 \mu \mathrm{s}$, the incident A0 mode does not arrive at the sensor. As indicated in Fig. 12(d), the reflected S0 mode from the right end of the delamination completely surpasses the reflected A0 mode from its left end. This reflected S0 mode has already passed through the sensor position. From Fig. 13, the arrival time of the reflected wave from the delamination is identified as around $275 \mu \mathrm{s}$. The reflected wave actually arrives at the sensor between the time points shown in Figs. 12(c) and 12(d), i.e., $236 \mu$ s and $303 \mu$ s. Therefore, the reflected wave signal detected clearly by the sensor is considered to be the reflected S0 mode from the right end rather than the left end of the beam, due to its higher intensity. Based on the numerical simulations above, the very complex interaction between the different signal modes and the boundaries of delamination can be identified. When only a single S0 mode passes through the delamination, four modes will be generated at one end of the delamination, including two transmitted A0 and S0 


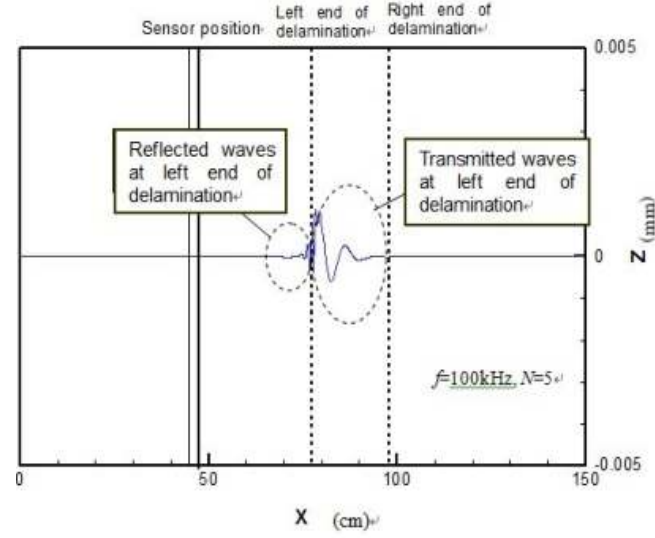

(a) $139 \mu \mathrm{s}$

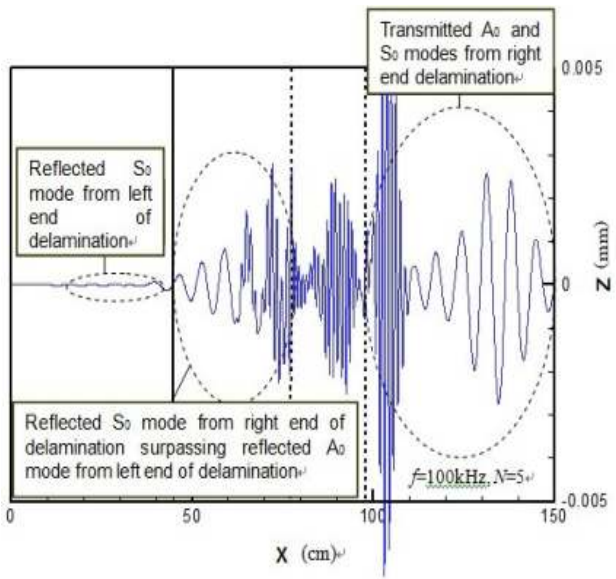

(c) $236 \mu \mathrm{s}$

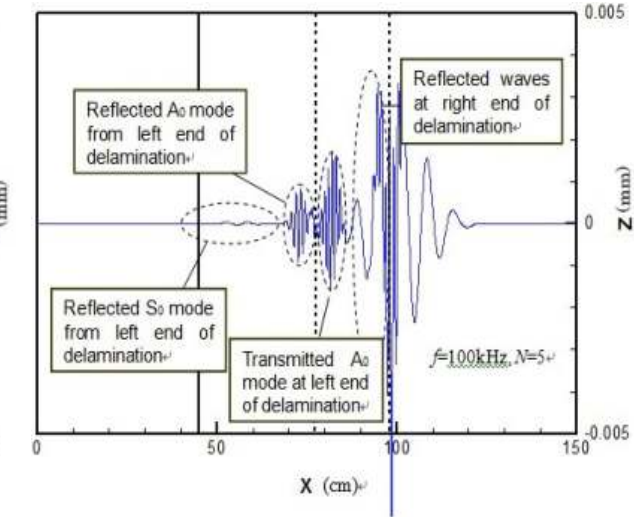

(b) $182 \mu \mathrm{s}$

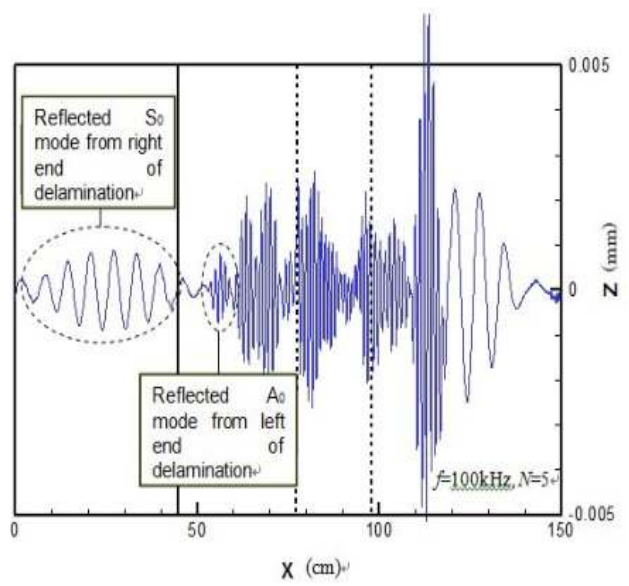

(d) $303 \mu \mathrm{s}$

Figure 12. Wave signal differences of intact and delaminated beams(S0 mode) 
modes, and two reflected A0 and S0 modes. Further study is required to understand why the stronger reflected $\mathrm{A} 0$ and $\mathrm{S} 0$ modes. However, the reflected $\mathrm{S} 0$ mode received by a sensor is from the reflections are from the right end of delamination. One possible explanation is that the delaminated region is of lower bending stiffness, which can be considered to be a softer region. When a wave propagates from a harder or intact region into a softer region, the reflections become weak. In contrast, when the wave propagates from a softer region into a harder one, a stronger reflection is expected.

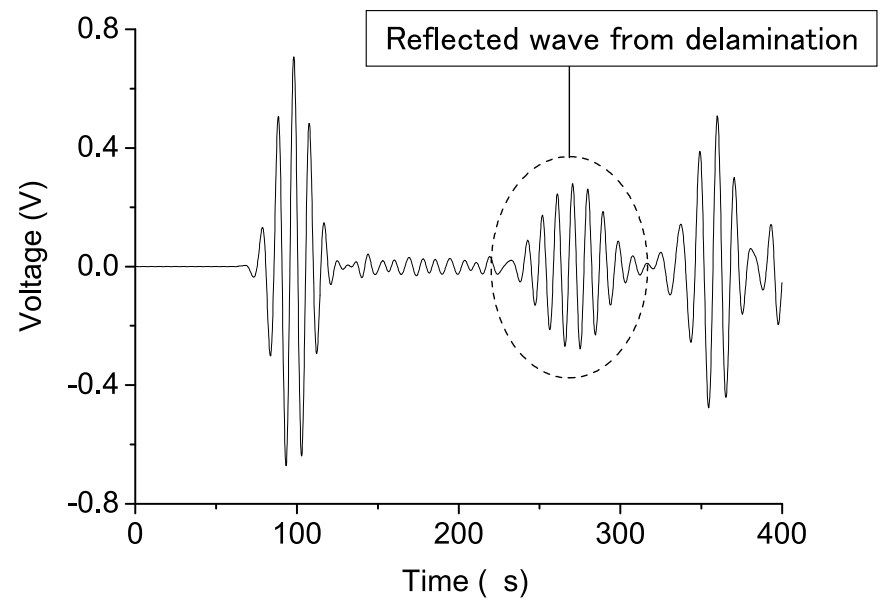

Figure 13. Sensor signal for a delamination of length of $200 \mathrm{~mm}$

\subsection{A0 mode}

Like S0 mode, a beam with a length of $1200 \mathrm{~mm}$ and a $200 \mathrm{~mm}$ long delamination case was examined using the numerical simulation under a condition of $f=50 \mathrm{kHz}$ and $N=5$. The transverse deflections (out-of-plane deformation) at various mesh points on the top surface of the beam were used. Fig. 14 also shows the subtraction between the wave signals from the intact and the delaminated beams, which can amplify the scattered wave signals from the delamination. The typical sensor signal for accurately evaluating the arrival times of various waves is shown in Fig. 15 with an enlarged picture for reflected waves from the delamination.

Firstly, for the laminate with $\left[010 / / 90_{6} / 90_{6} / 0_{10}\right]$, at the time domain of $318 \mu \mathrm{s}$, two A0 mode waves were excited by the actuator pair, which propagates to the left and right directions, independently. The A0 mode wave propagating to the right direction arrives at the left end of the delamination and then reflects from or transmits from the left end, as shown in Fig. 14(a), respectively. At $433 \mu \mathrm{s}$, the transmitted A0 mode from the left end of delamination passes through the right end. Fig. 14(b) shows the reflected wave from the right end of delamination. By comparing Fig. 14(b) and Fig. 15 at the same time domains, we can identify that the reflected A0 mode from the left end of delamination passes through the sensor. The reflected S0 mode due to mode change can also be identified. In principle, it is easy to 


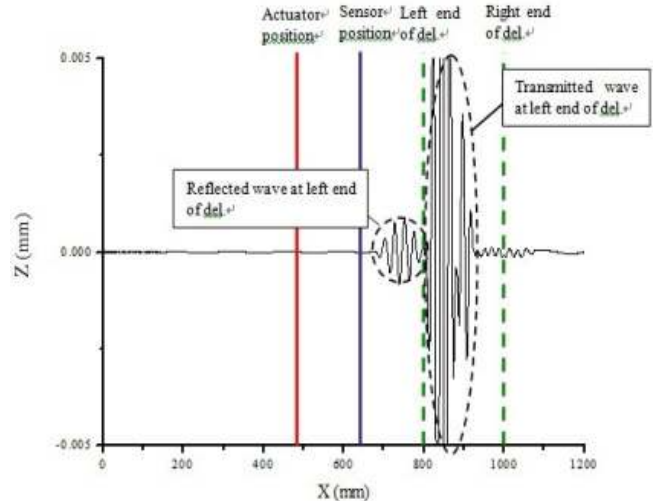

(a) $318 \mu \mathrm{s}$

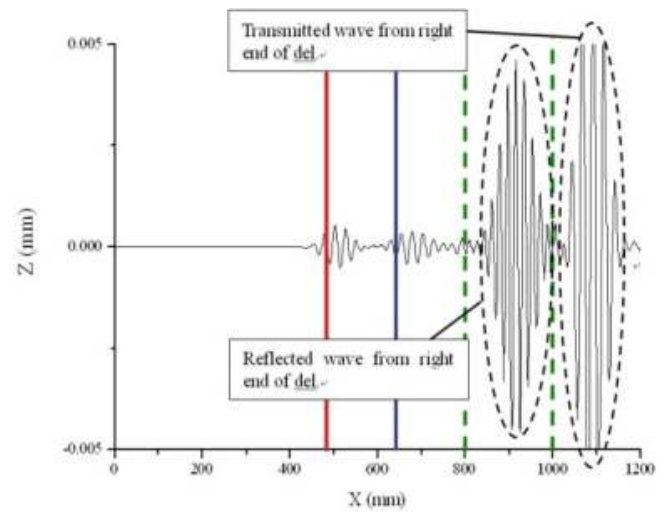

(c) $448 \mu \mathrm{s}$

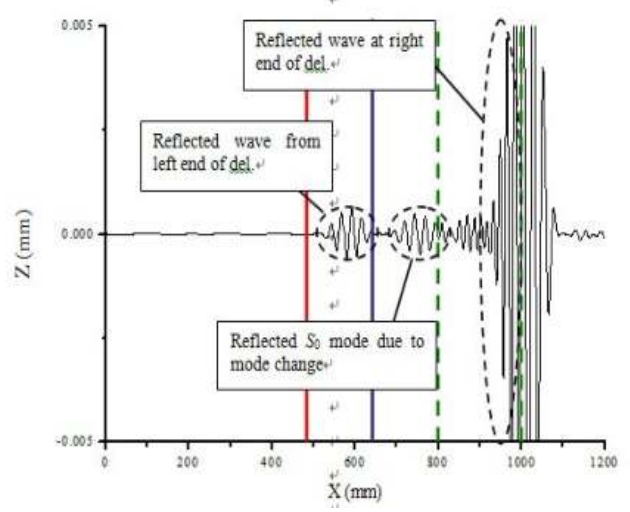

(b) $433 \mu \mathrm{s}$

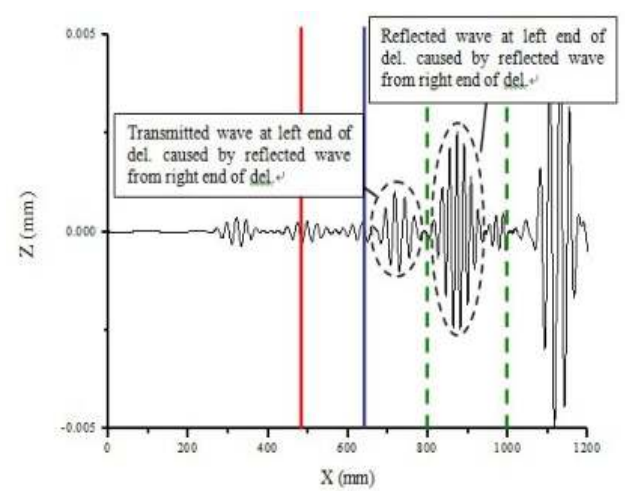

(d) $621 \mu \mathrm{s}$

Figure 14. Wave signal differences of intact and delaminated beams(A0 mode)

distinguish a S0 from an A0 mode by using phase information of signals from the two sensors. At $488 \mu \mathrm{s}$, the reflected and transmitted waves at the right end of the delamination separate to each other, as shown in Figs. 14(c). Also, the amplitude of the reflected wave is much higher than that of the reflected wave at the left end of the delamination, confirmed in Fig. 14(b). This phenomenon is similar to that obtained for S0 mode. Fig. 14(d) shows the transmitted and reflected waves at the left end of the delamination when the reflected wave from the right end of the delamination arrives at the left end at $621 \mu \mathrm{s}$. It is worthwhile mentioning that although the amplitude of reflected wave from the right end of delamination is very strong, the reflection at another end (left end) of the delamination can significantly reduce the amplitude of the transmitted wave, which is expected to be monitored by the sensors. Therefore, wave mode change and multiple reflections may create complicated interactions between the A0 mode and the delamination. 

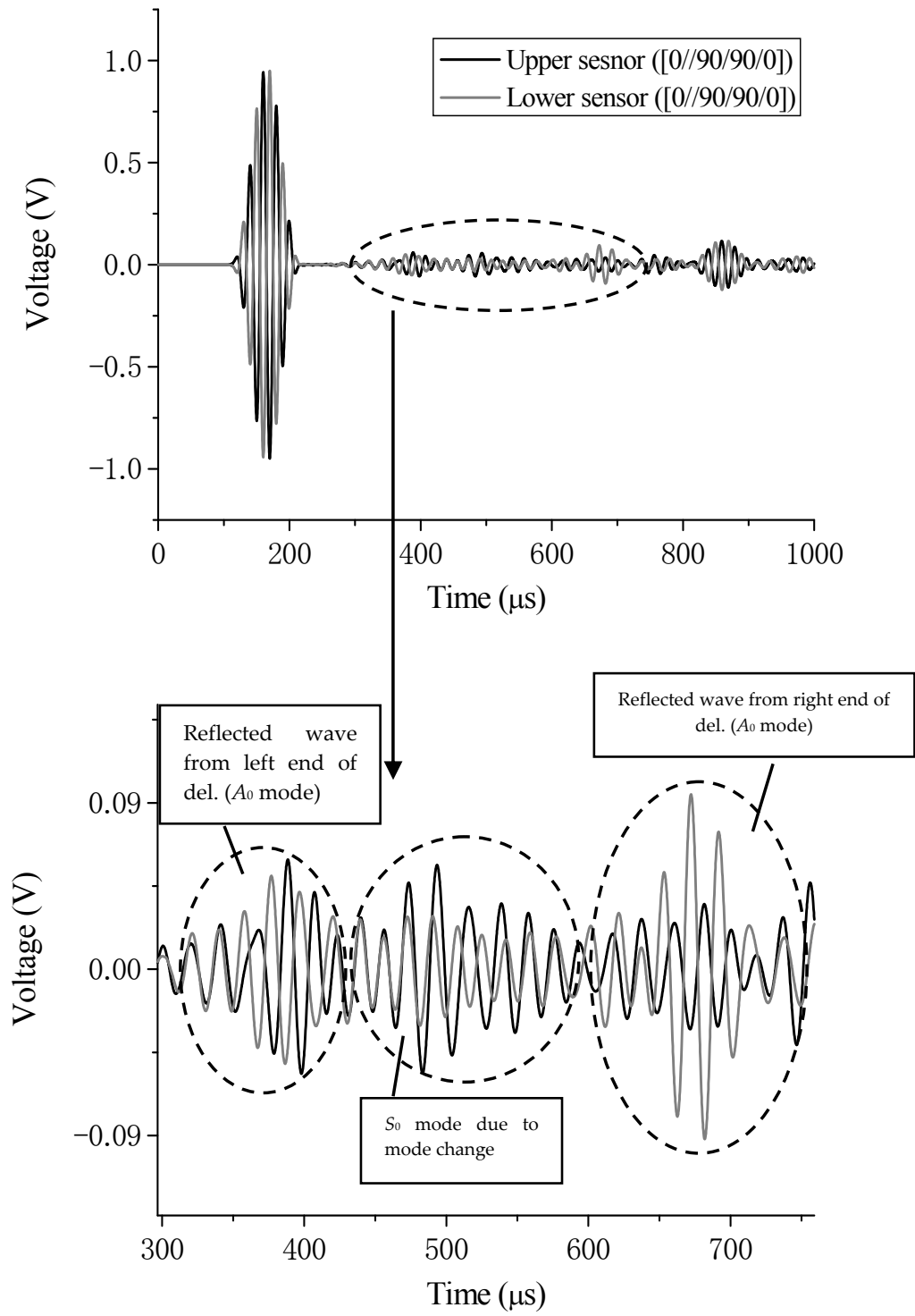

Figure 15. Sensor signal for delamination of $200 \mathrm{~mm}$ in length $\left(\left[0{ }_{10} / / 90_{6} / 90_{6} / 0_{10}\right]\right)$ 


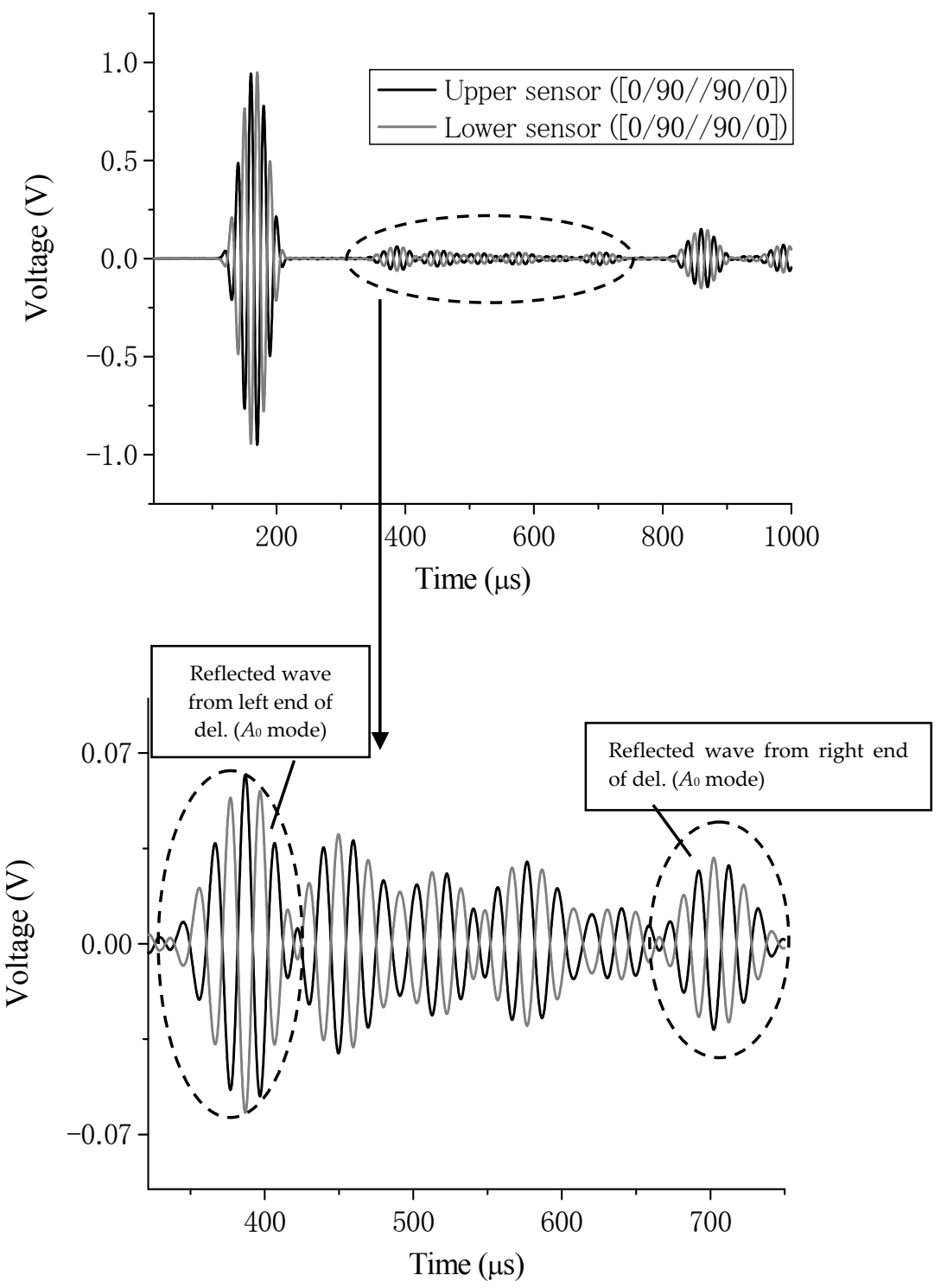

Figure 16. Sensor signal for delamination of $200 \mathrm{~mm}$ in length $\left(\left[00_{10} / 90_{6} / / 90_{6} / 0_{10}\right]\right)$ 
In Fig. 15, the amplitude of the reflected wave from the right end of the delamination is still higher than that of the reflected wave from the left end. Similar results can be observed in the laminate with $\left[0_{12} / / 0_{4} / 0_{4} / 0_{12}\right]$. In consequence, as shown in Table 2, more identified delamination positions are close to the right end of delamination for the laminates with $\left[0_{10} / / 90_{6} / 90_{6} / 0_{10}\right]$ and $\left[0_{12} / / 0_{4} / 0_{4} / 0_{12}\right]$. This phenomenon is similar to the result obtained for S0 mode in the case of $\left[010 / / 90_{6} / 90_{6} / 0_{10}\right]$.

For the case of $\left[0_{10} / 90_{6} / / 90_{6} / 0_{10}\right]$, the signals of two sensors are plotted in Fig. 16 with the enlarged picture for the reflection from the delamination. In Fig. 16, there is no S0 mode and the mode change does not occur as the delamination is located on the mid-plane of the laminates. In contrast to the cases of $\left[0_{10} / / 90_{6} / 90_{6} / 0_{10}\right]$ and $\left[0_{12} / / 0_{4} / 0_{4} / 0_{12}\right]$, it is interesting to note that the amplitude of the reflected wave from the left end of delamination is higher than that of the right end. Similar results are obtained in the case of $\left[0_{12} / 0_{4} / / 0_{4} / 0_{12}\right]$. Corresponding to Table 2, more identified delamination positions are close to the left end of the delamination. The physical meaning of why the stronger reflection occurs at the different ends of delamination for different delamination cases in Figs 15 and 16 is not clear. The position of the stronger reflection seems to mainly depend on the following two factors: bending stiffness of upper and lower delaminated layers and their difference, and Lamb wave deformation mode.

\section{Conclusion}

A technique for identifying a delamination damage in cross-ply laminated composite beams has been developed by using the zero-order mode of Lame waves. The delamination position can be identified if the arrival time of the arrival time of a reflected wave from a delamination and the wave propagation speed of Lame waves are known. One of the advantages of this approach is the baseline data acquired from intact beams are not required.

And, extensive finite element simulations have been conducted to investigate the interaction of Lame waves with various delamination cases. For S0 mode, when a single S0 mode propagates into a delamination, mode change happens and reflected S0 mode with high amplitude is generated from the end point of the delamination where the waves propagate out; for A0 mode, it has been found that the situation is more complex than $\mathrm{S} 0$ mode. For the laminates with stack sequence of $\left[0_{10} / / 9_{6} / 90_{6} / 0_{10}\right]$ and $\left[0_{12} / / 0_{4} / 0_{4} / 0_{12},\right]$, mode change also happens and the reflected wave from the right end of delamination is slight higher than that from the left end. However, for the case of $\left[0_{10} / 9_{6} / / 0_{6} / 0_{10}\right]$ and $\left[0_{12} / 0_{4} / / 0_{4} / 0_{12}\right]$, there is no mode change when a single A0 mode passes through delamination, and the stronger reflection from the delamination occurs at the left end of the delamination.

\section{Author details}

Yaolu Liu, Alamusi, Jinhua Li, Huiming Ning, Liangke Wu and Ning $\mathrm{Hu}$ Department of Mechanical Engineering, Chiba University, Yayoi-cho, Inage-ku, Chiba, Japan 
Weifeng Yuan and Bin Gu

School of Manufacturing Science and Engineering,

Southwest University of Science and Technology, Mianyang, P.R.China

\section{References}

[1] Su ZQ, Ye L, Lu Y. Guided Lamb waves for identification of damage in composite structures: A review. J Sound \& Vibr 2006; 295: 753-80.

[2] Valdes SHD, Soutis C. A structural health monitoring system for laminated composite. Proceedings of DETC, Pittsburgh, PA, USA; 2001.

[3] Sohn H, Farrar CR. Damage diagnosis using time series analysis of vibration signals. Smart Mater and Struct 2001; 10: 1-6.

[4] Ihn JB, Chang FK. Built-in diagnostics for monitoring crack growth in aircraft structures. In: Chang Fu-Kuo (ed.), Proceedings of the international workshop on structural health monitoring. Stanford University, USA; 2001.

[5] Hurlesbaus S, Niethammer M, Jacobs LJ, Valle C. Automated methodology to locate notches with Lamb waves. Acoustics Research Letters Online 2001; 2: 97-102.

[6] Su Z, Yang C, Pan N, Ye L, Zhou LM. Assessment of delamination in composite beams using shear horizontal (SH) wave mode. Compos Sci Technol 2007; 67: 244-51.

[7] Guo N, Cawley P. The interaction of Lamb waves with delaminations in composite laminates. J Acoust Soc Am 1993; 94: 2240-6.

[8] Wang $\mathrm{CH}$, Rose LRF. Wave reflection and transmission in beams containing delamination and inhomogeneity. J Sound \& Vibration 2003; 264: 851-72.

[9] Mahapatra DR, Gopalakrishnan S. A spectral finite element model for analysis of axialflexural-shear coupled wave propagation in laminated composite beams. Compos Struct 2003; 59: 67-88.

[10] Cao YP, Hu N, Lu J, Fukunaga H, Yao ZH. A 3D brick element based on Hu-Washizu variational principle for mesh distorsion. Int J Numer Meth Engrg 2002; 53: 252948.

[11] $\mathrm{Hu} \mathrm{N}$, Sekine H, Fukunaga H, Yao ZH. Impact analysis of composite laminates with multiple delaminations. Int J Impact Eng 1999; 22: 633-48.

[12] $\mathrm{Hu} \mathrm{N}$, A solution method for dynamics contact problems. Comput E Struct 1997; 63: 1053-63.

[13] Li CF, Hu N, Cheng JG, Sekine H, Fukunaga H. Low-velocity impact-induced damage of continuous fiber-reinforced composite laminates. Part II. verification and numerical investigation, Compos part A 2002; 33: 1063-72.

[14] Jeong H, Jang YS. Wavelet analysis of plate wave propagation in composite laminates. Compos Struct 2000; 49: 443-50.

[15] Nayfeh AH, Chimenti DE. Elastic wave propagation in fluid-loaded multi-axial anisotropic media. J Acoust Soc Am 1991; 89: 542-9. 
[16] Toyama N, Noda J, Okabe T. Quantitative damage detection in cross-ply laminates using Lamb wave method. Compos Sci and Technol 2003; 63: 1473-79.

[17] Takeda N, Okabe Y, Kuwahara J, Kojima S, Ogisu T. Development of smart composite structures with small-diameter fiber Bragg grating sensors for damage detection: Quantitative evaluation of delamination length in CFRP laminates using Lamb wave sensing. Compos Sci Technol 2005; 65: 2575-87. 